Pneumologe 2009 · 6:106-110 DOI 10.1007/s10405-008-0297-6

Online publiziert: 20. Februar 2009

(c) Springer Medizin Verlag 2009

\section{Redaktion}

F.J. Meyer, Heidelberg

Th.E. Wessendorf, Essen
B. Hoksch $\cdot$ K. Wucherpfennig $\cdot$ R. Schmid

DMLL / Klinik und Poliklinik für Thoraxchirurgie, Inselspital, Universitätsklinik Bern, Schweiz

\title{
Zerebraler Krampfanfall und Lungenrundherd unter Immunsuppression
}

\section{Anamnese}

Der Patient, ein Landwirt, mit dem Erstereignis eines tonisch-klonischen Krampfanfalles, wurde notfallmäßig zur stationären Aufnahme zugewiesen. Anamnestisch zu erheben waren eine schwere COPD bei positiver Raucheranamnese sowie eine seit 8 Wochen bestehende immunsuppressive Therapie mit Azathioprin und Steroiden bei Pyoderma gangraenosum am linken Unterschenkel (nachgewiesen: Streptococcus pneumoniae ca. 3 Monate zuvor). Weitere Angaben waren ein ungewollter Gewichtsverlust von ca. 6-10 kg in den letzten 3 Wochen, kein Fieber, kein Nachtschweiß.

\section{Klinik}

Der noch immer stark adipöse 72-jährige Patient $(\mathrm{BMI}>40)$ präsentierte sich in einem sehr schlechten Allgemeinzustand bei ausgeprägter Komorbidität (neben der bereits erwähnten COPD bestanden eine schwere koronare und hypertensive Kardiopathie mit Status nach Vorderwandinfarkt sowie ein steroidinduzierter Diabetes mellitus).

Bei der klinischen Untersuchung auffällig waren ein chronischer, produktiver Husten (weißlich-gelber, teilweise auch brauner Auswurf), eine Belastungsdyspnoe NYHA II-III und Unterschenkelödeme beidseits mit einem $4 \times 3 \mathrm{~cm}$ großen, ca. $3 \mathrm{~mm}$ tiefen Ulkus am linken Unterschenkel. Auskultatorisch fand sich über beiden Pulmones ein exspiratorisches Giemen bei insgesamt gedämpftem Atemgeräusch. Die neurologische Untersuchung war ohne pathologische Befunde.

\section{Diagnostik}

Entzündungswerte. Entzündungswerte (unter immunsuppressiver Therapie) nur mäßig erhöht. Leukozyten: 16,3 G/l, CrP: $62 \mathrm{mg} / \mathrm{l}$.

Lungenfunktionsprüfung. Schwere obstruktive Ventilationsbehinderung bei $\mathrm{FEV}_{1}$ 0,98 1 (37\%), FVC 1,97 (57\%) und negativem Inhalationstest.

aBGA. Hypoxämie mit $\mathrm{pO}_{2}$ von $61,2 \mathrm{~mm}$ $\mathrm{Hg}$.

Bildgebende Diagnostik. Im CT Nachweis einer frontalen zerebralen Raumforderung links mit leichtem perifokalem Ödem (• Abb. 1). Thorax-CT (- Abb. 2). Im Kontroll-MRT des Schädels leichte Größenprogredienz des zerebralen Herdes um $1 \mathrm{~cm}$ auf 2,6 cm Durchmesser im Vergleich zum CT vor $21 \mathrm{Ta}$ gen sowie Zeichen einer möglichen Inflammation.

Bronchoskopie/Bürste/BAL. Acht Tage vor Zuweisung: unauffällig für maligne Zellen, unauffällige Mikroskopie. Im Tracheobronchialsekret Nachweis von Leukozyten und grampositiven Kokken (Kultur: Mischflora aus Moraxella catarrhalis, Streptococcus pneumoniae, Haemophilus influenzae).

Blutkulturen und Sputumproben. Es wurden Blutkulturen und Sputumproben zur mikrobiologischen Untersuchung gewonnen. 


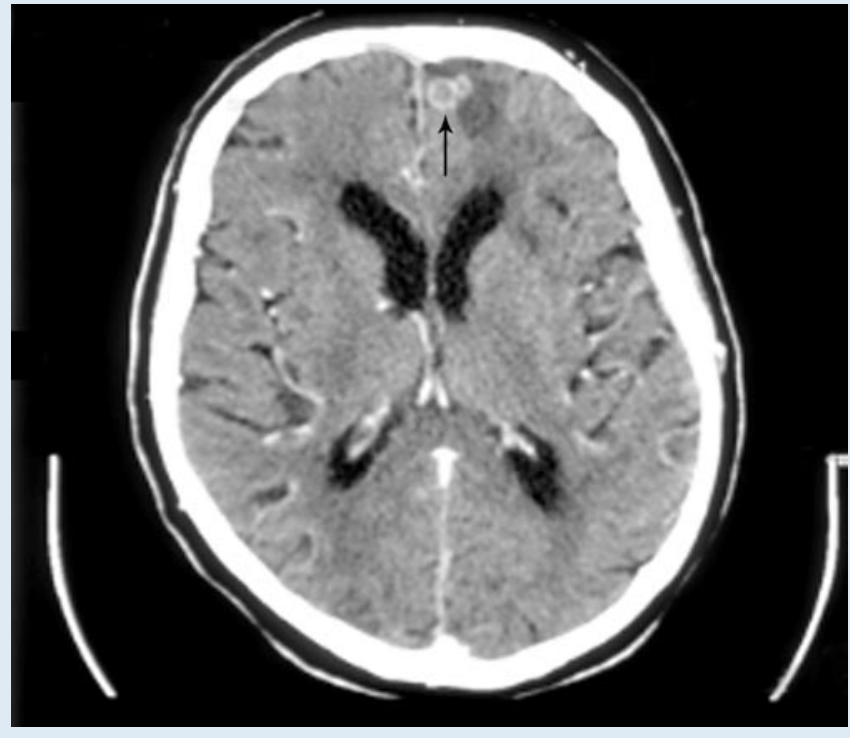

Abb. $1<$ Schädel-CT

mit Darstellung einer zerebralen Raumforderung links mit perifokalem Ödem

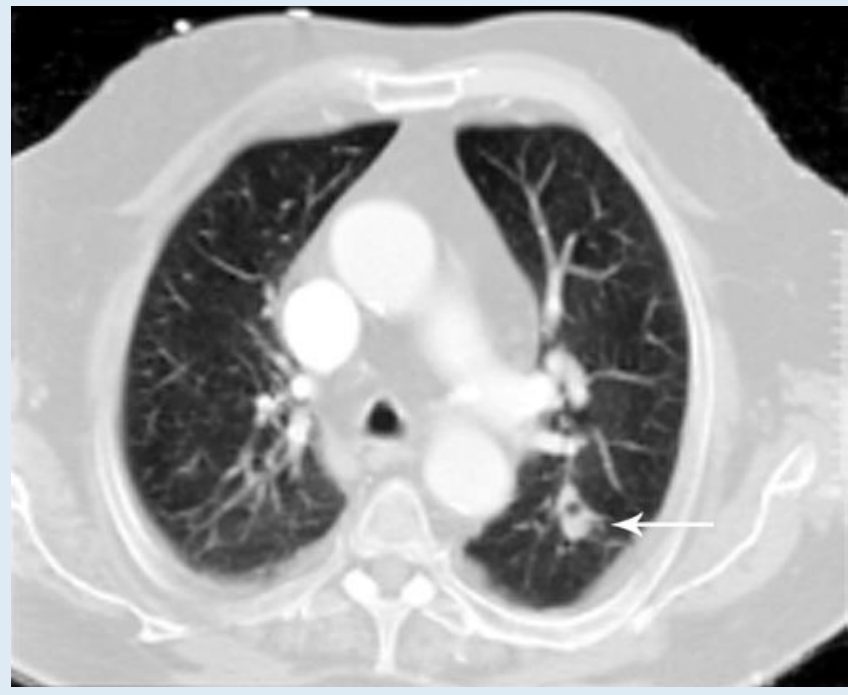

Abb. $2<$ Unklare pulmonale Raumforde-

rung links mit zentraler Einschmelzung (s. Pfeil) 
\ Diagnose: Systemische Nokardiose

\section{Definition}

Die Nokardiose stellt eine seltene grampositive bakterielle Infektion mit aeroben Aktinomyzeten der Gattung Nocardia dar. Nokardien - 22 Spezies sind heute bekannt - kommen ubiquitär vor: in Erdboden, Feuchtbiotopen, verfaulenden Früchten. Sie gelangen v. a. via Inhalation in den menschlichen Körper. Zwei Drittel der Infektionen mit Nokardien betreffen deshalb die Lunge. Verletzungen der Haut ermöglichen eine kutane Infektion [3]. Unterschieden wird die lokalisierte von der systemischen (disseminierten) Infektion. Bei Letzterer sind durch hämatogene Streuung 2 oder mehr Organsysteme, z. B. ZNS, Knochen, Retina, Herz, Nieren, betroffen. Die systemische Dissemination ist mit einer hohen Mortalität verbunden.

\section{Pathogenese}

Nokardiosen treten sowohl bei immunkompetenten als auch bei abwehrgeschwächten Patienten auf. Allerdings weist die überwiegende Mehrzahl der Patienten eine Immunsupprimierung verschiedenster Genese auf, z. B. im Rahmen der Transplantationschirurgie, bei Autoimmunerkrankungen, HIV oder chronischen Erkrankungen wie der COPD, insbesondere auf der zellvermittelten Ebene.

\section{(2) Diabetes, Alkoholmissbrauch und Tuberkulose können eine Nokardiose begünstigen}

Diabetes, Alkoholabusus und Tuberkulose sind weitere Faktoren, die eine Nokardiose begünstigen können $[2,4,6]$. Da die Erreger überall in der Natur vorkommen, ist anzunehmen, dass für Personen, die in der Landwirtschaft tätig sind und eine immunsuppressive Therapie erhalten, ein erhöhtes Risiko hinsichtlich der Nokardiose besteht.

\section{Diagnostik}

Da die klinische Symptomatik völlig unspezifisch ist (Fieber, allgemeine B-Symptomatik, Husten bei pulmonalem Befall), wird die Nokardiose oft nicht oder zu spät differenzialdiagnostisch in Erwägung gezogen. Somit vergeht in der Regel wertvolle Zeit bis zur Diagnosestellung - die Angaben hierzu schwanken in der Literatur zwischen 2 Tagen und 12 Monaten [1].

\section{- Differenzialdiagnostisch auszuschließen sind v. a. Infektionen mit Aspergillen, Mykobakterien und Kryptokokken.}

Bei den mikrobiologischen Anforderungen von z. B. Blut- und Sputumproben sollte deshalb - insbesondere bei prädisponierten Patienten - explizit die Untersuchung auf Nokardien verlangt werden, damit u. a. spezielle Kulturen angelegt werden und bestimmte Reagenzien, die Nokardien zerstören können (z. B. Cysteinverbindungen), nicht zum Einsatz kommen. Neben den üblichen Färbe- und Kulturtechniken hat insbesondere die PCR aufgrund von schnellen und sicheren Ergebnissen einen hohen Stellenwert. Untersuchungen mit der 16S-rRNA Sequence-Analyse zeigten eine 100\%ige Treffsicherheit für alle eingesandten Proben (Sputum, Abszessmaterial, Hautbiopsien; [5]).

\section{Therapie}

Neben einer u. U. im Einzelfall notwendigen chirurgischen Sanierung (z. B. bei Hirnabszessen) besteht die Therapie der Nokardiose in einer Langzeitantibiotikagabe, wobei systematische Untersuchungen zur Therapiedauer in der Literatur fehlen. Empfohlen werden eine 1- bis 3-monatige Therapie bei kutanem Befall, die Nokardiose der Lunge sollte 6 Monate und eine zerebrale Nokardiose 12 Monate behandelt werden.

\section{- Immunsupprimierte Patienten sollten generell mindestens 1 Jahr therapiert werden.}

Sulfonamide in Kombination mit Trimethoprin sind das am häufigsten zum Einsatz kommende Therapeutikum. Alternativen sind Augmentin-Clavulansäure, Amikacin, 3.-Generation-Cephalosporine oder Fluoroquinolone. Auch auf diesem Gebiet fehlen entsprechend prospektiv-randomisierte Studien, die Mono- und Kombinationstherapie der verschiedenen Antibiotika vergleichen. Das Problem bei einigen Nocardia-Spezies, wie z. B. Nocardia farcinica, stellt die relativ ausgeprägte Resistenz gegenüber zahlreichen, sonst gut wirksamen Antibiotika dar [1].

\section{Verlauf im vorliegenden Fall}

Im vorgestellten Fall verstarb der Patient 2 Monate nach Diagnosestellung. Die Obduktion ergab als Todesursache das Vorliegen einer Rechtsherzinsuffizienz mit multiplen beidseitigen parazentralen Lungenembolien. Des Weiteren lagen 2 chronische Hirnabszesse von 1,8 und o,6 cm Durchmesser vor. Ein Erregernachweis gelang in den Spezialfärbungen nicht.

\section{Prognose}

Insgesamt weist die disseminierte Nokardiose eine hohe Mortalität auf, von 7-44\% bei immunkompetenten bis über $85 \%$ bei immunsupprimierten Patienten sowie bei zerebralem oder disseminiertem Befall steigend [1].

\section{Fazit für die Praxis}

Bei einer steigenden Inzidenz von Patienten, die eine Immunsuppression aufweisen, sollten bei unklaren pulmonalen Prozessen mit oder ohne zerebrale Beteiligung neben dem Bronchialkarzinom auch systemische Infektionen $\mathbf{u}$. a. mit Nokardien abgeklärt werden. Eine entsprechende Anforderung muss dazu vom behandelnden Arzt an das mikrobiologische Referenzlabor begleitend zu den eingesandten Blut- und Sputumproben gehen. 


\section{Korrespondenzadresse}

PD Dr. B. Hoksch

DMLL / Klinik und Poliklinik für
Thoraxchirurgie, Inselspital,
Universitätsklinik Bern
3010 Bern
Schweiz
beatrix.hoksch@insel.ch

Interessenkonflikt. Die korrespondierende Autorin gibt an, dass kein Interessenkonflikt besteht.

\section{Literatur}

1. Agertof $A J$, van der Bruggen $T$, Tersmette $M$ et al (2007) Nocardiosis: a case series and a mini review of clinical and microbiological features. Neth J Med 6:199-202

2. Lederman ER, Crum NF (2004) A case series and focused review of nocardiosis: clinical and microbiologic aspects. Medicine 83:300-313

3. Safdar N, Kaul DR, Saint S (2007) Into the woods. N Engl J Med 9:943-947

4. Tomas RM, Villanueva RM, Calzada SR et al (2007) Pulmonary nocardiosis: risk factors and outcomes. Respirology 12:394-400

5. Wauters G, Avesani V, Charlier J et al (2005) Distribution of nocardia species in clinical samples and their routine rapid identification in the laboratory. J Clin Microbiol 43:2624-2628

6. Wilson JP, Turner HR, Kirchner KA, Chapman SW (1989) Nocardial infections in renal transplant recipients. Medicine (Baltimore) 68:38-57
Pneumologe 2009 · 6:106-110 DOI 10.1007/s10405-008-0297-6

๑) Springer Medizin Verlag 2009

\section{B. Hoksch $\cdot$ K. Wucherpfennig $\cdot$ R. Schmid Zerebraler Krampfanfall und Lungenrundherd unter Immunsuppression}

\section{Zusammenfassung}

Die Zahl der Patienten, die eine immunsuppressive Therapie erhalten, ist steigend. Demzufolge sollte bei unklaren pulmonalen Prozessen mit oder ohne zerebrale Beteiligung neben einem Bronchialkarzinom immer auch an eine opportunistische systemische Infektion gedacht werden. Als ein Beispiel dafür wird hier über eine systemische Nokardiose berichtet. Da die klinische Symptomatik der Nokardiose völlig unspezifisch ist, wird sie oft nicht oder zu spät differenzialdiagnostisch in Erwägung gezogen. Nokardien kommen ubiquitär vor. Sie gelangen v. a. via Inhalation

in den menschlichen Körper. Unterschieden wird die lokalisierte von der systemischen (disseminierten) Infektion, die mit einer hohen Mortalität, insbesondere bei immunsupprimierten Patienten, verbunden ist. In der Regel besteht die Therapie der Nokardiose in einer Langzeitantibiotikagabe.

\section{Schlüsselwörter}

Nokardiose - Nokardien · Immunsuppression . Opportunistische Infektion · Lungenrundherd

\section{Cerebral seizure and pulmonary nodule under immunosuppression}

\section{Abstract}

The number of patients undergoing immunosuppressive therapy is increasing. Thus in patients with unknown pulmonary processes with or without cerebral involvement, not only lung cancer but also opportunistic systemic infection should be included in the differential diagnosis. A case study of systemic nocardiosis is presented by way of example. Since the clinical signs of nocardiosis are nonspecific, the disease is often not taken into consideration until a very late stage, if at all. Nocardias are found ubiquitously. Inha- lation is the most common mode of entry. A distinction must be made between a localised infection and a systemic (disseminated) one. Systemic infection is associated with a high mortality rate, especially in immunosuppressed patients. As a general rule, longterm administration of antibiotics is the treatment of choice.

Keywords Nocardiosis · Nocardia · Immunosuppression . Opportunistic infection · Lung nodule

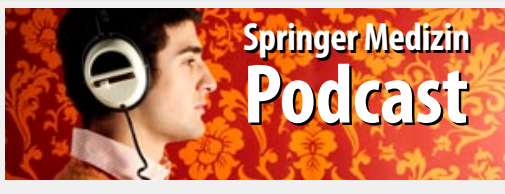

- Kongressnews

- Spannendes aus der Welt der Medizin - Interviews

Jeden Monat neu!

Jetzt kostenlos downloaden unter

www.springer.de/podcast 


\section{Hier steht eine Anzeige.}

Springer 